\title{
Correction to: Group leader emotional intelligence and group performance: a multilevel perspective
}

\author{
Yucheng Zhang ${ }^{1}$ - Long Zhang ${ }^{2} \mathbb{D}$ - Jingtao Zhu ${ }^{3} \cdot$ Chih-Hsing Liu ${ }^{4}$. \\ Mengxi Yang ${ }^{5} \cdot$ Guangjian Liu $^{6}$
}

Published online: 14 August 2020

๑) Springer Nature Limited 2020

\section{Correction to: Asian Business \& Management https://doi.org/10.1057/s41291-020-00123-1}

In the original publication of the article, the affiliation of the author Mengxi Yang was incorrect. The correct affiliation should be as given below:

School of Economics and Management, University of Chinese Academy of Sciences, Beijing, China.

Further, the author biography should read as given below:

The original article can be found online at https://doi.org/10.1057/s41291-020-00123-1.

Long Zhang

lyon.long.zhang@gmail.com

Yucheng Zhang

yucheng.eason.zhang@gmail.com

Jingtao Zhu

david.jingtao.zhu@gmail.com

Chih-Hsing Liu

phd20110909@gmail.com

Mengxi Yang

mengxi.yang2014@qq.com

Guangjian Liu

lgj19890622@126.com

1 School of Economics and Management, Hebei University of Technology, Tianjin, China

2 Business School, Hunan University, Changsha, China

3 AIESEC in Mainland of China, Beijing, China

4 Ming Chuan University, Taipei, Taiwan

5 School of Economics and Management, University of Chinese Academy of Sciences, Beijing, China

6 Department of Organization and Human Resources, School of Business, Renmin University of China, Beijing, China 
Mengxi Yang (PhD, Tsinghua University) is an Assistant Professor at School of Economics and Management, University of Chinese Academy of Sciences, Beijing, China. His research interest lies in leadership and human resource management.

The original article has been updated accordingly.

Publisher's Note Springer Nature remains neutral with regard to jurisdictional claims in published maps and institutional affiliations. 